\title{
INTEGRAL IDENTITIES OF NORMS AND CHARACTERIZATION OF INNER PRODUCT SPACES
}

\author{
ČASLAV V. STANOJEVIĆ AND ANA M. SUCHANEK
}

ABSTRACT. Integral identities of norms over compact groups are used to characterize inner product spaces. These integral identities also generalize the PenicoStanojevic [1] integral form of the parallelogram law.

1. Recently Penico and Stanojević [1] proved that a normed complex linear space $N$ is an inner product space if and only if

$$
\frac{1}{2 \pi} \int_{0}^{2 \pi}\|(\cos t) x+(\sin t) y\|^{2} d t<1
$$

for all unit vectors $x$ and $y$ in $N$. This characterization is an integral analogue to the Schoenberg [2] version of the classical M. M. Day [3] result.

In this paper we shall consider a more general type of integral of norms than the one in the prior inequality, and show that certain integral identities characterize inner product spaces among normed linear spaces.

2. Throughout the paper $N$ will denote a normed complex linear space, $C$ the field of complex numbers and $\Gamma=\{z|| z \mid=1\}$, the unit circle of $C$.

In the next theorem we shall give necessary and sufficient conditions for $N$ to be an inner product space in terms of norm integrals over compact groups.

THEOREM. Let $G$ be a compact topological group with normalized Haar measure $\mu$, and let $\gamma \neq 1$ be a continuous homomorphism of $G$ into $\Gamma$. Then $N$ is an inner product space if and only if

$$
\int_{G}\|x+\gamma(g) y\|^{2} d \mu=\|x\|^{2}+\|y\|^{2}
$$

for all $x, y \in N$.

Proof. The "only if" part. Notice that from

$$
\int_{G} \gamma(g) d \mu=\int_{G} \gamma(a g) d \mu=\gamma(a) \int_{G} \gamma(g) d \mu
$$

it follows that

$$
\int_{G} \gamma(g) d \mu=0
$$

for $\gamma \not 1$.

Received by the editors January 8, 1980.

AMS (MOS) subject classifications (1970). Primary 46C10.

$K e y$ words and phrases. Inner product spaces. 
Assume that $N$ is an inner product space. Then

$$
\int_{G}\|x+\gamma(g) y\|^{2} d \mu=\|x\|^{2}+\|y\|^{2}+\int_{G} \operatorname{Re}[\overline{\gamma(g)}(x, y)] d \mu,
$$

where $(\cdot, \cdot)$ is the inner product in $N$. The integral on the right-hand side is zero because of $(\dagger)$. Hence $(*)$ follows.

The "if" part. Any complex two-dimensional subspace $N_{2}$ of $N$ can be considered as a real four-dimensional space, and the unit sphere $S_{2}$ of $N_{2}$, as a central convex body in that real four-dimensional space. Let $E$ be an ellipsoid inscribed in $S_{2}$ and having maximal four-dimensional volume. Then, from a result of Dvoretzky and Rogers [4], it follows that there are two complex linearly independent vectors $x$ and $y$ in $E \cap S_{2}$.

The ellipsoid $E$ induces a euclidean norm $\|\cdot\|_{E}$ on $N_{2}$, and $E$ is the unit sphere of $N_{2}$ with respect to $\|\cdot\|_{E}$. Thus

$$
\|x\|=\|y\|=\|x\|_{E}=\|y\|_{E}=1 .
$$

Since $E \subset S_{2}$, we have that $\|u\|<\|u\|_{E}$ for all $u \in N_{2}$.

Let $x$ and $y$ be the previous complex linearly independent vectors, and let $\alpha$ and $\beta$ be any two complex numbers. Since $N_{2}$ is an inner product space with respect to $\|\cdot\|_{E}$, it follows that (*) holds for any $\alpha x, \beta y \in N_{2}$, i.e.

$$
\int_{G}\|\alpha x+\gamma(g) \beta y\|_{E}^{2} d \mu=\|\alpha x\|_{E}^{2}+\|\beta y\|_{E}^{2}=|\alpha|^{2}+|\beta|^{2}
$$

Assume (*) and recall that $\|\cdot\| \leqslant\|\cdot\|_{E}$. Then

$$
\begin{aligned}
|\alpha|^{2}+|\beta|^{2} & =\|\alpha x\|^{2}+\|\beta y\|^{2}=\int_{G}\|\alpha x+\gamma(g) \beta y\|^{2} d \mu \\
& \leqslant \int_{G}\|\alpha x+\gamma(g) \beta y\|_{E}^{2} d \mu=|\alpha|^{2}+|\beta|^{2} .
\end{aligned}
$$

Hence

$$
\int_{G}\left(\|\alpha x+\gamma(g) \beta y\|_{E}^{2}-\|\alpha x+\gamma(g) \beta y\|^{2}\right) d \mu=0
$$

Since for every open subset $U$ of $G, \mu(U)>0$, and since the integrand of the last integral is a nonnegative continuous function in $g$, we have that

$$
\|\alpha x+\gamma(g) \beta y\|_{E}=\|\alpha x+\gamma(g) \beta y\|
$$

for all $g \in G$. In particular for $g=e,\|\alpha x+\beta y\|_{E}=\|\alpha x+\beta y\|$ for all $\alpha, \beta \in C$.

Every vector $u \in N_{2}$ is a linear combination of $x$ and $y$, i.e. $u=\alpha x+\beta y$. Hence we proved that $\|u\|_{E}=\|u\|$ for all $u \in N_{2}$.

From the standard result it follows that $N$ is an inner product space. This completes the proof of the theorem.

3. The scope of our theorem is best viewed through the following examples.

Example 1. Let $G=\mathbf{R} / 2 \pi Z$, with the quotient topology $\gamma(t)=e^{i t}$ and let $\mu$ be the Lebesgue measure on $G$. Then $N$ is an inner product space if and only if 


$$
\int_{G}\left\|x+e^{i t} y\right\|^{2} d t=\frac{1}{2 \pi} \int_{0}^{2 \pi}\left\|x+e^{i t} y\right\|^{2} d t=\|x\|^{2}+\|y\|^{2},
$$

for all $x, y \in N$.

Example 2. Let $\mu$ be the normalized arc length on $\Gamma$. On the $n$-dimensional torus $G=\Pi_{j=1}^{n} \Gamma_{j}$, for each $n$-tuple $n=\left(m_{1}, \ldots, m_{n}\right)$ of integers, the function

$$
\gamma_{m}\left(z_{1}, \ldots, z_{n}\right)=\prod_{j=1}^{n} z_{j}^{m}
$$

is a continuous homomorphism from $G$ into $C$. Then $N$ is an inner product space if and only if

$$
\int_{\Gamma} \int_{\Gamma} \ldots \int_{\Gamma}\left\|x+\left(\prod_{j=1}^{n} z_{j}^{m_{j}}\right) y\right\|^{2}\left|d z_{1}\right|\left|d z_{2}\right| \cdots\left|d z_{n}\right|=\|x\|^{2}+\|y\|^{2}
$$

for all $x, y \in N$.

In particular we have that $N$ is an inner product space if and only if

$$
\int_{\Gamma}\left\|x+z^{n} y\right\|^{2}|d z|=\|x\|^{2}+\|y\|^{2}
$$

for all $x, y \in N$.

Example 3. A normed complex linear space $N$ is an inner product space if and only if

$$
\frac{1}{(2 \pi)^{n}} \int_{0}^{2 \pi} \int_{0}^{2 \pi} \cdots \int_{0}^{2 \pi}\left\|\sum_{k=1}^{n} e^{i t_{k}} x_{k}\right\|^{2} d t_{1} d t_{2} \ldots d t_{n}=\sum_{k=1}^{n}\left\|x_{k}\right\|^{2}
$$

for all $x_{k} \in N$.

\section{REFERENCES}

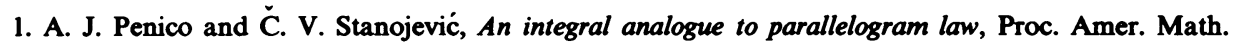
Soc. 79 (1980), 427-430.

2. I. J. Schoenberg, A remark on M. M. Day's characterization of inner-product spaces and a conjecture of L. M. Blumenthal, Proc. Amer. Math. Soc. 3 (1952), 361-364.

3. M. M. Day, Some characterizations of inner-product spaces, Trans. Amer. Math. Soc. 82 (1947), 320-337.

4. A. Dvoretzky and C. A. Rogers, Absolute and conditional convergence in normed linear spaces, Proc. Nat. Acad. Sci. U.S.A. 36 (1950), 192-197.

Department of Mathematics, University of Missouri-Rolla, Rolla, Missouri 65401 